\title{
Statistics on partitions arising from seaweed algebras
}

\author{
Vincent E. Coll Jr. \\ Department of Mathematics \\ Lehigh University \\ Bethlehem, U.S.A. \\ vec208@lehigh.edu
}

\author{
Andrew W. Mayers \\ Sceptor \\ State College, U.S.A. \\ seaweed@amayers.us
}

\author{
Nicholas W. Mayers \\ Department of Mathematics \\ Lehigh University \\ Bethlehem, U.S.A.
}

nwm5095@gmail.com

Submitted: Mar 8, 2020; Accepted: Jun 19, 2020; Published: Jul 10, 2020

(c) The authors. Released under the CC BY license (International 4.0).

\begin{abstract}
Using the index theory of seaweed algebras, we explore various new partition statistics. We find relations to some well-known families of partitions as well as a surprising periodicity result.
\end{abstract}

Mathematics Subject Classifications: 05A17, 17B99

\section{Introduction}

Comment: This paper may be regarded as complimentary to a recent paper by Seo and Yee [24]. Their paper is based on a prior arXiv [14] version of this paper, where the notion of partition statistics based on seaweed algebras is introduced.

Partition statistics are often defined with an eye toward proving a congruence property. An application of this principal can be found in the proofs of the famous congruence results of Ramanujan [23], which were eventually established using the rank and crank statistics $[3,5]$. On the other hand, a partition statistic may present itself without prior appeal to an anticipated congruence property. The recent index theory of seaweed algebras $[10,11,12,15]$ provides just such an instance. Indeed, seaweed subalgebras of $\mathfrak{s l}(n)-$ or simply, seaweeds - which are naturally defined in terms of two compositions of a single integer $n$, provide a sort of partition statistic generator.

One begins with a pair $(\lambda, \mu)$ with $\lambda$ a partition and $\mu$ a composition of $n$. Since partitions are compositions, we can use $(\lambda, \mu)$ to define a seaweed subalgebra of $\mathfrak{s l}(n)$, whose index (a non-negative integer) will be taken to be the definition of the index of the pair $(\lambda, \mu)$. If $\mu$ is a " $\lambda$-based" composition $\mu(\lambda)$, then the index of the pair $(\lambda, \mu(\lambda))$ may be regarded as a partition statistic on $\lambda$ alone. Letting $w(\lambda)$ denote the weight of $\lambda$, there are two choices of $\mu$ naturally associated with a given $\lambda$, namely, the trivial partition where $\mu=w(\lambda)$, and its conjugate $\mu^{C}=1^{w(\lambda)}$. It is remarkable that this pedestrian definition 
of an index-based statistic is of double-edged utility, yielding insights into two ostensibly unrelated theories: the classical study of bijections between restricted partitions and the more topical study of the enumeration of certain important classes of seaweed algebras.

In the first case, $\left(\lambda, 1^{w(\lambda)}\right)$, we find a connection to classical partition theory. If $\mathcal{P}(n)$ is the set of partitions of weight $n$, then the sequence $\left\{c_{n}^{i}\right\}_{n=1}^{\infty}$ defined by

$$
c_{n}^{i}=\left|\left\{\lambda \in \mathcal{P}(n): \operatorname{ind}_{1^{w(\lambda)}}(\lambda)=n-i\right\}\right|,
$$

for each fixed $i$ is eventually constant - stabilizing at the number of two-colored partitions of $i-1$ (see Theorem 11).

In the second case, we consider seaweeds defined by the pair $(\lambda, w(\lambda))$. The enumeration of such composition pairs, when the index is zero, is of concern to Lie theorists. ${ }^{1}$ Recent efforts to enumerate pairs of compositions that define a Frobenius (index zero) seaweed have concentrated on limiting the number of parts in the compositions. For example, Duflo and $\mathrm{Yu}$ use certain index-preserving operators on the set of compositions corresponding to a Frobenius seaweed subalgebra of $\mathfrak{s l}(n)$ to show that if $t$ is the number of parts in the defining compositions, then the number of such compositions is a rational polynomial of degree $\left[\frac{t}{2}\right]$ evaluated at $n$ (see [17], Theorem 1.1 (b)). Duflo and Yu's result is existential in nature. However, if compositions are restricted to partitions and a modest limit is placed on the size of the parts - rather than the number of parts - the number of such compositions corresponding to a Frobenius seaweed subalgebra of $\mathfrak{s l}(n)$ becomes a periodic function of $n$ (see Theorem 16).

The organization of the paper is as follows. In Section 2 we develop the definitions and notation for partitions and seaweeds. In Section 3 we use the index theory of seaweeds to define index-based statistics on partitions and use this new construction to connect to some well-known classical investigations. We conclude with some open questions and directions for further study.

\section{Preliminaries}

In Section 2.1 we review standard combinatorial notation. In Section 2.2 we detail the recent index theory of seaweed algebras. Throughout this article, we tacitly assume that all Lie algebras are over the complex numbers.

\subsection{Partitions}

We follow the notation of Andrews [1] and adopt the following conventions.

Definition 1. A partition $\lambda$ of a positive integer $n$ is a finite non-increasing sequence of positive integers $\lambda_{1}, \lambda_{2}, \ldots, \lambda_{m}$ such that $n=\sum_{i=1}^{m} \lambda_{i}$. The $\lambda_{i}$ are called the parts of the partition and $w(\lambda)=n$ is the weight of the partition.

\footnotetext{
${ }^{1}$ Frobenius algebras form a distinguished class and have been extensively studied from the point of view of invariant theory [22] and are of special interest in deformation and quantum group theory resulting from their connection with the classical Yang-Baxter equation (see [19] and [20]).
} 
We will often employ the vector notation for the partition $\lambda=\left(\lambda_{1}, \lambda_{2}, \ldots, \lambda_{m}\right)$. It will sometimes be useful to use a frequency notation that makes explicit the number of times a particular integer occurs as a part of a partition. So, if $\lambda=\left(\lambda_{1}, \lambda_{2}, \ldots, \lambda_{m}\right)$, we alternatively write

$$
\lambda=\left(1^{f_{1}} 2^{f_{2}} 3^{f_{3}} \cdots\right),
$$

where exactly $f_{i}$ of the $\lambda_{j}$ are equal to $i$.

A graphical representation of a partition, called a Ferrers diagram, is helpful to develop the notion of the conjugate of a partition. More formally, the Ferrers diagram of a partition $\lambda=\left(\lambda_{1}, \ldots, \lambda_{m}\right)$ is a coordinatized set of unit squares in the plane such that the lower left corner of each square will have integer coordinates $(i, j)$ such that

$$
0 \geqslant i \geqslant-m+1,0 \leqslant j \leqslant \lambda_{|i|+1}-1 .
$$

The conjugate of a partition $\lambda$ is the partition $\lambda^{C}$ resulting from exchanging the rows and columns in the Ferrers diagram associated to $\lambda$. The Ferrers diagram of the partition $(4,2,1)$, as well as its conjugate $(3,2,1,1)$, are illustrated in Figure 1.
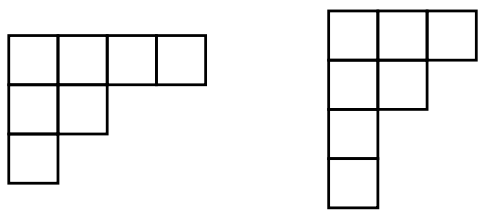

Figure 1: Ferrers Diagram of $(4,2,1)$ and $(3,2,1,1)$

\subsection{Seaweed algebras}

In this section, we introduce seaweed algebras in $\mathfrak{s l}(n)$ - the set of all $n \times n$ matrices of trace zero. As we will see, such seaweed algebras are naturally defined in terms of two compositions of the positive integer $n$. Recall that a composition of $n$ is an unordered partition, which we will denote by $\lambda_{1}\left|\lambda_{2}\right| \cdots \mid \lambda_{m}$ to distinguish it from the ordered case in Definition 1, where there is an order relation on the $\lambda_{i}^{\prime} s$.

Definition 2. If $V$ is an $n$-dimensional vector space with a basis $\left\{e_{1}, \ldots, e_{n}\right\}$, let $a_{1}|\cdots| a_{m}$ and $b_{1}|\cdots| b_{t}$ be two compositions of $n$ and consider the flags

$$
\{0\} \subset V_{1} \subset \cdots \subset V_{m-1} \subset V_{m}=V \quad \text { and } \quad V=W_{0} \supset W_{1} \supset \cdots \supset W_{t}=\{0\},
$$

where $V_{i}=\operatorname{span}\left\{e_{1}, \ldots, e_{a_{1}+\cdots+a_{i}}\right\}$ and $W_{j}=\operatorname{span}\left\{e_{b_{1}+\cdots+b_{j}+1}, \ldots, e_{n}\right\}$.

The subalgebra of $\mathfrak{s l}(n)$ preserving these flags is called a seaweed Lie algebra, or simply seaweed, and is denoted by the symbol $\frac{a_{1}|\cdots| a_{m}}{b_{1}|\cdots| b_{t}}$, which we refer to as the type of the seaweed. If $b_{1}=n$, the seaweed is called maximal parabolic. 
Remark 3. The preservation of flags in Definition 2 insures that seaweeds are closed under matrix multiplication, and therefore define an associative algebra, hence also a Lie algebra under the commutator bracket.

The evocative "seaweed" is descriptive of the shape of the algebra when exhibited in matrix form. For example, the seaweed algebra $\frac{2 \mid 4}{1|2| 3}$ consists of traceless matrices of the form depicted on the left side of Figure 2, where *'s indicate potential non-zero entries.
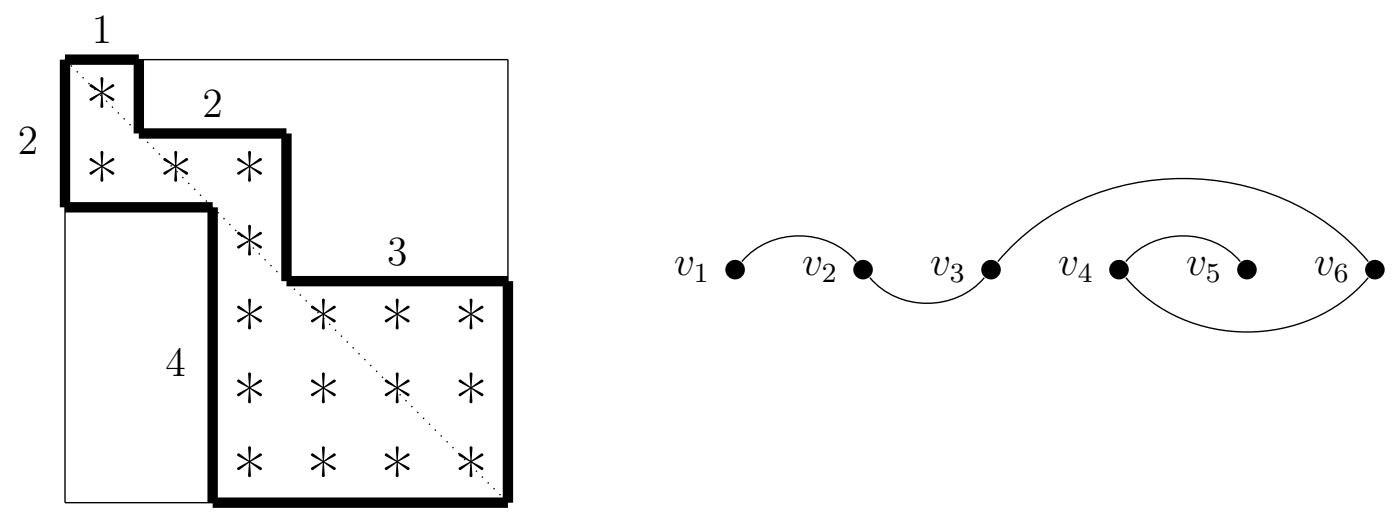

Figure 2: A seaweed of type $\frac{2 \mid 4}{1|2| 3}$ and its associated meander

The index of a Lie algebra was introduced by Dixmier [16]. Formally, the index of a Lie algebra $\mathfrak{g}$ is defined by

$$
\operatorname{ind}(\mathfrak{g})=\min _{f \in \mathfrak{g}^{*}} \operatorname{dim}\left(\operatorname{ker}\left(B_{f}\right)\right)
$$

where $B_{f}$ is the associated skew-symmetric Kirillov form defined by $B_{f}(x, y)=f([x, y])$, for all $x, y \in \mathfrak{g}$. The index is an important algebraic invariant of the Lie algebra though notoriously difficult to compute. However, in [15], Dergachev and A. Kirillov developed a combinatorial algorithm to compute the index of a seaweed subalgebra of $\mathfrak{s l}(n)$ by counting the number of connected components of a certain planar graph, called a meander, associated to the seaweed.

To construct a meander, let $\frac{a_{1}|\cdots| a_{m}}{b_{1}|\cdots| b_{t}}$ be a seaweed. Now label the $n$ vertices of our meander as $v_{1}, v_{2}, \ldots, v_{n}$ from left to right along a horizontal line. We then place edges above the horizontal line, called top edges, according to $a_{1}+\cdots+a_{m}$ as follows: Partition the set of vertices into a set partition by grouping together the first $a_{1}$ vertices, then the next $a_{2}$ vertices, and so on, lastly grouping together the final $a_{m}$ vertices. We call each set within a set partition a block. For each block in the set partition determined by $a_{1}+\cdots+a_{m}$, add an edge from the first vertex of the block to the last vertex of the block, then add an edge between the second vertex of the block and the second to last vertex of the block, and so on within each block. More explicitly, given vertices $v_{j}, v_{k}$ in a block of size $a_{i}$, there is an edge between them if and only if $j+k=2\left(a_{1}+a_{2}+\cdots+a_{i-1}\right)+a_{i}+1$. 
In the same way, place bottom edges below the horizontal line of vertices according to the blocks in the partition determined by $b_{1}+\cdots+b_{t}$ (see the right side of Figure 2).

Every meander consists of a disjoint union of cycles and paths. The main result of [15] is that the index of a seaweed can be computed by counting the number and type of these components in its associated meander.

Theorem 4. (Dergachev and A. Kirillov, [15]) If $\mathfrak{p}$ is a seaweed subalgebra of $\mathfrak{s l}(n)$, then

$$
\operatorname{ind}(\mathfrak{p})=2 C+P-1 \text {, }
$$

where $C$ is the number of cycles and $P$ is the number of paths in the associated meander.

Example 5. In the example of Figure 2, the meander associated to the seaweed $\frac{2 \mid 4}{1|2| 3}$ has no cycles and consists of a single path - so, has index zero, hence is Frobenius.

While Theorem 4 is an elegant combinatorial result it is difficult to apply in practice. However, Coll et al in [13] show that any meander can be contracted or "wound-down" to the empty meander through a sequence of graph-theoretic moves, each of which is uniquely determined by the structure of the meander at the time of move application. There are five such moves, only one of which affects the component structure of the meander graph and is therefore the only move capable of modifying the index of the meander. Since we will need the explicit winding-down moves in the proof of Theorem 16 we review the winding-down process.

Lemma 6 (Winding-down). Given a meander $M$ of type $\frac{a_{1}\left|a_{2}\right| \cdots \mid a_{m}}{b_{1}\left|b_{2}\right| \cdots \mid b_{t}}$, create a meander $M^{\prime}$ by exactly one of the following moves.

1. Vertical Flip $\left(\boldsymbol{F}_{\boldsymbol{v}}\right)$ : If $a_{1}<b_{1}$, then $M^{\prime}$ has type $\frac{b_{1}\left|b_{2}\right| \cdots \mid b_{t}}{a_{1}\left|a_{2}\right| \cdots \mid a_{m}}$.

2. Component Elimination $(\boldsymbol{C}(\boldsymbol{c}))$ : If $a_{1}=b_{1}=c$, then $M^{\prime}$ has type $\frac{a_{2}\left|a_{3}\right| \cdots \mid a_{m}}{b_{2}\left|b_{3}\right| \cdots \mid b_{t}}$.

3. Rotation Contraction $(\boldsymbol{R})$ : If $b_{1}<a_{1}<2 b_{1}$, then $M^{\prime}$ has type

$$
\frac{b_{1}\left|a_{2}\right| a_{3}|\cdots| a_{m}}{\left(2 b_{1}-a_{1}\right)\left|b_{2}\right| \cdots \mid b_{t}} .
$$

4. Block Elimination $(\boldsymbol{B})$ : If $a_{1}=2 b_{1}$, then $M^{\prime}$ has type $\frac{b_{1}\left|a_{2}\right| \cdots \mid a_{m}}{b_{2}\left|b_{3}\right| \cdots \mid b_{t}}$.

5. Pure Contraction $(\boldsymbol{P})$ : If $a_{1}>2 b_{1}$, then $M^{\prime}$ has type $\frac{\left(a_{1}-2 b_{1}\right)\left|b_{1}\right| a_{2}\left|a_{3}\right| \cdots \mid a_{m}}{b_{2}\left|b_{3}\right| \cdots \mid b_{t}}$. For all moves, except the Component Elimination move, $M$ and $M^{\prime}$ have the same index. 
Example 7. In this example, the seaweed $\frac{17 \mid 3}{10|4| 6}$ is wound-down to the empty meander using the moves detailed in Lemma 6.

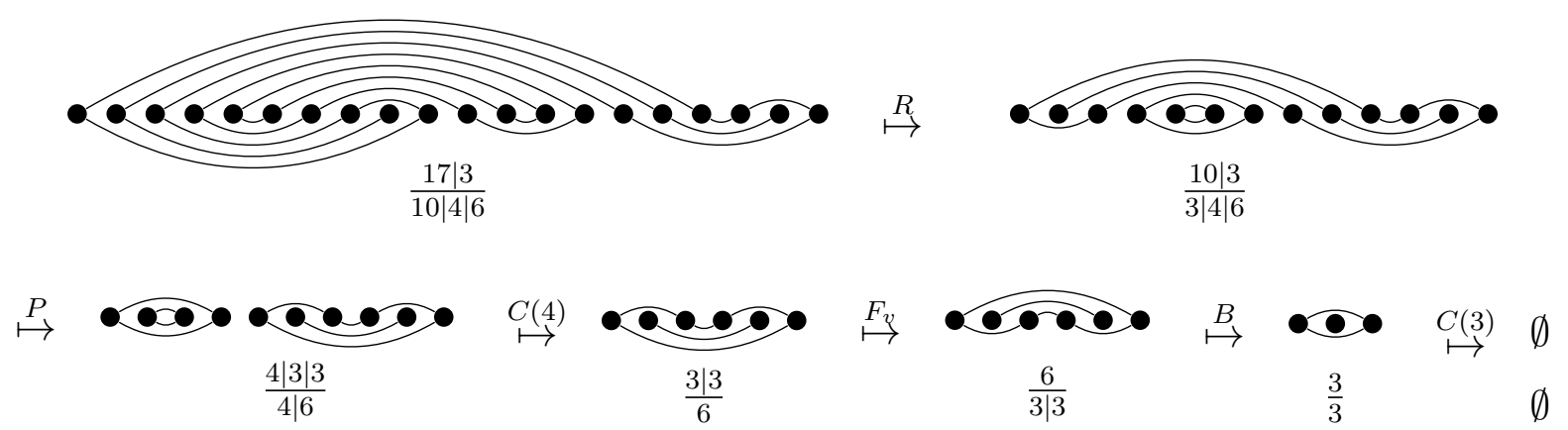

Figure 3: Winding down the meander $\frac{17 \mid 3}{10|4| 6}$

In what follows, it is helpful to add a sixth index preserving transformation, $F_{h}$, called a horizontal flip which takes $M$ to $\frac{a_{m}|\cdots| a_{2} \mid a_{1}}{b_{t}|\cdots| b_{2} \mid b_{1}}$.

\section{The index of a partition}

Let $\lambda=\left(\lambda_{1}, \lambda_{2}, \ldots, \lambda_{m}\right)$ be a partition and $\mu=\left(\mu_{1}, \mu_{2}, \ldots, \mu_{t}\right)$ a composition of $n$. These compositions can be used to define the seaweed

$$
\mathfrak{p}(\lambda, \mu)=\frac{\lambda_{1}\left|\lambda_{2}\right| \cdots \mid \lambda_{m}}{\mu_{1}\left|\mu_{2}\right| \cdots \mid \mu_{t}}
$$

Now, define the index of the pair $(\lambda, \mu)$ to be the index of $\mathfrak{p}(\lambda, \mu)$, and write $\operatorname{ind}_{\mu}(\lambda)$. If $\mu$ is a $\lambda$-based composition $\mu(\lambda)$, then $\operatorname{ind}_{\mu(\lambda)}(\lambda)$ may be regarded as a partition statistic on $\lambda$ alone.

There are two choices for $\mu$ naturally associated with $\lambda$, namely, $w(\lambda)$ and its conjugate $1^{w(\lambda)}$. These yield, respectively, two partition statistics on $\lambda$ defined as follows:

$$
\operatorname{ind}_{w(\lambda)}(\lambda)=\operatorname{ind}\left(\frac{\lambda_{1}|\cdots| \lambda_{m}}{w(\lambda)}\right) \quad \text { and } \quad \operatorname{ind}_{1 w(\lambda)}(\lambda)=\operatorname{ind}\left(\frac{\lambda_{1}|\cdots| \lambda_{m}}{1|\cdots| 1}\right) .
$$

Example 8. Let $\lambda=(3,2,1)$ in (1). An application of Theorem 3 now yields

$$
\operatorname{ind}_{6}(\lambda)=\operatorname{ind}\left(\frac{3|2| 1}{6}\right)=0 \quad \text { and } \quad \operatorname{ind}_{1^{6}}(\lambda)=\operatorname{ind}\left(\frac{3|2| 1}{1|1| 1|1| 1 \mid 1}\right)=3 \text {. }
$$




\section{$3.1\left(\lambda, 1^{w(\lambda)}\right)$}

In this section we investigate, for fixed $i$ and varying $n$, the sequence of values defined by the number of partitions $\lambda \in \mathcal{P}(n)$ such that $\operatorname{ind}_{1 w(\lambda)}(\lambda)=n-i$. We find that for each $i$, if $c_{n}^{i}=\left|\left\{\lambda \in \mathcal{P}(n): \operatorname{ind}_{1 w(\lambda)}(\lambda)=n-i\right\}\right|$, then $\left\{c_{n}^{i}\right\}_{n=i}^{\infty}$ is eventually constant, stabilizing at $c^{i}$, which turns out to be a well-known classical value (see Theorem 11). The following Table 1 illustrates $c_{n}^{i}$ for small values of $n$ and $i$.

\begin{tabular}{|c|c|c|c|c|c|c|c|c|c|c|}
\hline $\boldsymbol{n} \backslash \boldsymbol{i}$ & $\mathbf{0}$ & $\mathbf{1}$ & $\mathbf{2}$ & $\mathbf{3}$ & $\mathbf{4}$ & $\mathbf{5}$ & $\mathbf{6}$ & $\mathbf{7}$ & $\mathbf{8}$ & $\mathbf{9}$ \\
\hline $\mathbf{1}$ & 1 & 0 & 0 & 0 & 0 & 0 & 0 & 0 & 0 & 0 \\
\hline $\mathbf{2}$ & 1 & 1 & 0 & 0 & 0 & 0 & 0 & 0 & 0 & 0 \\
\hline $\mathbf{3}$ & 0 & 2 & 1 & 0 & 0 & 0 & 0 & 0 & 0 & 0 \\
\hline $\mathbf{4}$ & 0 & 2 & 2 & 1 & 0 & 0 & 0 & 0 & 0 & 0 \\
\hline $\mathbf{5}$ & 0 & 0 & 4 & 2 & 1 & 0 & 0 & 0 & 0 & 0 \\
\hline $\mathbf{6}$ & 0 & 0 & 3 & 5 & 2 & 1 & 0 & 0 & 0 & 0 \\
\hline $\mathbf{7}$ & 0 & 0 & 0 & 7 & 5 & 2 & 1 & 0 & 0 & 0 \\
\hline $\mathbf{8}$ & 0 & 0 & 0 & 5 & 9 & 5 & 2 & 1 & 0 & 0 \\
\hline $\mathbf{9}$ & 0 & 0 & 0 & 0 & 12 & 10 & 5 & 2 & 1 & 0 \\
\hline $\mathbf{1 0}$ & 0 & 0 & 0 & 0 & 7 & 17 & 10 & 5 & 2 & 1 \\
\hline
\end{tabular}

Table 1: Number of $\lambda \in \mathcal{P}(n)$ with $\operatorname{ind}_{1 w(\lambda)}(\lambda)=i$.

By coloring partitions, we can better understand the $c^{i}$ s. We will use two colors (red and blue), to color the parts of a given partition. When enumerating colored partitions, we will assume that two partitions which are identical, save for their coloring, will be considered different partitions. So, for example, the partition of the integer 2 given by $(1,1)$ is different from the partition of the integer 2 given by $(1,1)$. We also tacitly assume that in a given colored partition all blue parts of a given size precede all red parts of the same size (see Example 9). Such colored partitions are called two-colored partitions.

Example 9. The two-colored partitions of 2 are: (2), (2), $(1,1),(1,1)$, and $(1,1)$.

Remark 10. Two-colored partitions make an appearance in Guptas's 1958 article [21] and have recently been connected to other diverse objects, such as quandles $[8,9]$.

The generating function for the number of two-colored partitions of $n$ is well-known and is given by

$$
\prod_{m \geqslant 1} \frac{1}{\left(1-x^{m}\right)^{2}} .
$$

The following theorem connects the current exposition to classical partition theory.

Theorem 11.

$$
\sum_{i \geqslant 1} c^{i} x^{i-1}=\prod_{m \geqslant 1} \frac{1}{\left(1-x^{m}\right)^{2}}
$$


Proof. The case $i=1$ is clear since, by Theorem 4 , the only $\lambda \in \mathcal{P}(n)$ with $\operatorname{ind}_{1^{w(\lambda)}}(\lambda)=$ $n-1$ is $\lambda=1^{n}$. We show that for $i>1$ and $n \geqslant 3 i-3$, there is a bijective correspondence between $\mathcal{M}(i, n)=\left\{\lambda \in \mathcal{P}(n): \operatorname{ind}_{1 w(\lambda)}(\lambda)=n-i\right\}$ and $\mathcal{P}^{2}(i-1)=$ $\{$ two-colored partitions of $i-1\}$. We do this in two steps.

First, we show that, for each $i>1$, the values $|\mathcal{M}(i, n)|=c_{n}^{i}$ do indeed stabilize to a fixed value $c^{i}$, for $n \geqslant 3 i-3$. Take $\lambda=\left(\lambda_{1}, \ldots, \lambda_{t}\right) \in \mathcal{M}(i, n)$. By Theorem 4 , we know that

$$
\operatorname{ind}_{1^{n}}(\lambda)=2 C+P-1=n-i .
$$

Since there are no bottom edges in the meander corresponding to $\mathfrak{p}\left(\lambda, 1^{n}\right)$, the meander contains no cycles nor paths of length greater than two. Thus, $\operatorname{ind}_{1^{n}}(\lambda)$ corresponds to one less than the number of top edges and isolated vertices. Evidently, each part $\lambda_{j}$ of $\lambda$ contributes $\left\lceil\lambda_{j} / 2\right\rceil$ to the number of edges and isolated vertices. Thus,

$$
\operatorname{ind}_{1^{n}}(\lambda)=\sum_{j=1}^{t}\left\lceil\frac{\lambda_{j}}{2}\right\rceil-1=n-i .
$$

After substituting $\sum_{j=1}^{t} \lambda_{j}$ for $n$, we find that

$$
\sum_{j=1}^{t}\left\lfloor\frac{\lambda_{j}}{2}\right\rfloor=i-1 .
$$

Equation (2) implies that $\lambda \in \mathcal{M}(i, n)$ if and only if the meander corresponding to $\mathfrak{p}\left(\lambda, 1^{n}\right)$ consists of $n$ vertices and $i-1$ top arcs. Therefore, ignoring parts of size one provides an injective map

$$
\psi: \mathcal{M}(i, n) \rightarrow \mathcal{M}(i-1),
$$

where $\mathcal{M}(i-1)$ denotes the set of partitions $\mu=\left(\mu_{1}, \ldots, \mu_{m}\right)$ such that $\mu_{m}>1$ and the meander corresponding to $\mathfrak{p}\left(\mu, 1^{w(\mu)}\right)$ has $i-1$ top arcs. Since the largest partition of $\mathcal{M}(i-1)$ has weight $3 i-3$, we may conclude that $\psi$ is a bijection, for $n \geqslant 3 i-3$; that is, the values $c_{n}^{i}$ stabilize at $c^{i}=|\mathcal{M}(i-1)|$, for $n \geqslant 3 i-3$.

Finally, we establish the generating function for $c^{i}$. To this end, we construct a bijection $\varphi: \mathcal{P}^{2}(i-1) \rightarrow \mathcal{M}(i-1)$. Let $\sigma=\left(\sigma_{1}, \ldots, \sigma_{m}\right) \in \mathcal{P}^{2}(i-1)$. Define $\varphi$ by setting $\varphi(\sigma)=\left(\mu_{1}, \ldots, \mu_{m}\right)$, where

$$
\mu_{i}= \begin{cases}2 \sigma_{i}+1, & \sigma_{i} \text { is blue } \\ 2 \sigma_{i}, & \sigma_{i} \text { is red }\end{cases}
$$

By construction, it is clear that $\varphi(\sigma) \in \mathcal{M}(i-1)$. Furthermore, $\varphi$ is invertible, so that $\varphi$ establishes a bijection between $\mathcal{P}^{2}(i-1)$ and $\mathcal{M}(i-1)$. Thus, $\varphi^{-1} \circ \psi: \mathcal{M}(i, n) \rightarrow \mathcal{P}^{2}(i-1)$ provides a bijection, for $n \geqslant 3 i-3$. The result follows.

Remark 12. In [24], Seo and Yee provide an alternative proof of Theorem 11 which relies on generating functions. 


\section{$3.2(\lambda, w(\lambda))$ - the maximal parabolic case}

As above, let $\lambda=\left(\lambda_{1}, \ldots, \lambda_{m}\right)$ be an element of $\mathcal{P}(n)$. In this section, we consider the seaweed defined by the pair of compositions $(\lambda, w(\lambda))$. In contrast to the previous section, here we investigate the number of partitions $\lambda$ such that $\operatorname{ind}_{w(\lambda)}(\lambda)=0$. We naturally call such partitions, Frobenius partitions. The main theorem of this section, Theorem 16 and Remark 17, remarkably establish that if $\lambda_{i} \leqslant 7$, for $i=1, \ldots, m$, then the number of Frobenius partitions is eventually a periodic function of $n$.

We begin with three Lemmas which will be helpful in the proof of Theorem 16 .

Lemma 13. Let $\mathfrak{g}=\frac{a_{1}|\cdots| a_{m}}{\sum_{i=1}^{m} a_{i}}$ be a seaweed algebra. If there exists $i<j-1$ such that $\sum_{l=1}^{i} a_{l}=\sum_{l=j}^{m} a_{l}$, then $\mathfrak{g}$ is not Frobenius.

Proof. Applying the winding moves $\left(F_{v}\right)$ followed by $i$ applications of $(P)$ to the meander corresponding to $\mathfrak{g}$ results in the meander corresponding to the seaweed algebra of type $\frac{b_{1}\left|a_{i}\right| \cdots \mid a_{1}}{a_{i+1}|\cdots| a_{j}|\cdots| a_{m}}$ where $b_{1}=\sum_{l=i+1}^{j-1} a_{l}>0$; but this meander consists of at least two components, one corresponding to $\frac{b_{1}}{a_{i+1}|\cdots| a_{j-1}}$, and the other $\frac{a_{i}|\cdots| a_{1}}{a_{j}|\cdots| a_{m}}$. Thus, by Theorem 4 , $\operatorname{ind}(\mathfrak{g})>0$.

Lemma 14. Let $\mathfrak{g}=\frac{a_{1}|\cdots| a_{m}}{\sum_{i=1}^{m} a_{i}}$ be a seaweed algebra. If there exists more than two odd $a_{i}$ 's, then $\mathfrak{g}$ is not Frobenius.

Proof. Each odd $a_{i}$ contributes a vertex of degree 1 to the meander corresponding to $\mathfrak{g}$. Furthermore, each open path consists of exactly two vertices of degree 1, and no closed path contains a vertex of degree 1 . So, if there are more than two odd $a_{i}$ 's, then the corresponding meander must contain more than one open path. Therefore, by Theorem 4 , $\operatorname{ind}(\mathfrak{g})>0$.

Lemma 15 (Theorem 10, [12]). If $a$ is even and $\operatorname{gcd}(a, b)=1$, then the seaweed of type $\frac{a|a| \cdots|a| b}{a+a+\cdots+a+b}$ is Frobenius.

Let $\mathcal{P}_{0}(n, d)$ be the set of Frobenius partitions $\lambda=\left(\lambda_{1}, \ldots, \lambda_{m}\right) \in \mathcal{P}(n)$ such that $\lambda_{i} \leqslant d$ for $1 \leqslant i \leqslant m$.

Theorem 16. If $d \in\{1,2,3,4\}$, then the values of $\left|\mathcal{P}_{0}(n, d)\right|$ are eventually periodic. More precisely,

- If $n \geqslant 3,\left|\mathcal{P}_{0}(n, 1)\right|=0$.

- If $n \geqslant 5$,

$$
\left|\mathcal{P}_{0}(n, 2)\right|= \begin{cases}1, & n \text { odd } \\ 0, & n \text { even }\end{cases}
$$

- If $n \geqslant 13$,

$$
\left|\mathcal{P}_{0}(n, 3)\right|= \begin{cases}2, & n \text { odd } \\ 0, & n \text { even }\end{cases}
$$


- If $n \geqslant 17$,

$$
\left|\mathcal{P}_{0}(n, 4)\right|= \begin{cases}4, & n \equiv 1(\bmod 4) ; \\ 2, & n \equiv 2(\bmod 4) ; \\ 3, & n \equiv 3(\bmod 4) ; \\ 0, & n \equiv 0(\bmod 4) .\end{cases}
$$

Proof. The proof heuristic is described as follows. We consider the possible partitions for each $d \leqslant 4$ - except for those cases considered in Lemma 13 and Lemma 14 - and determine which partitions are Frobenius.

$\mathbf{d}=1: \operatorname{ind}\left(\frac{1|\cdots| 1}{n}\right)=\left\lfloor\frac{n}{2}\right\rfloor>0$ for $n \geqslant 3$. Thus, for $n \geqslant 3$ we have $\left|\mathcal{P}_{0}(n, 1)\right|=0$.

$\mathbf{d}=\mathbf{2}, \mathbf{n} \geqslant \mathbf{5}$ : Using the results determined for the case $d=1$, we consider only partitions with $\lambda_{1}=2$. After applying Lemma 13 and Lemma 14, the only partitions remaining are those of the form $\left(1^{1} 2^{f_{2}}\right)$, which are Frobenius by Lemma 15 . Thus, there is exactly one such Frobenius partition if and only if the weight is odd.

$\mathbf{d}=\mathbf{3}, \mathbf{n} \geqslant 13$ : As before, using the results for the cases $d=1$ and $d=2$, we can restrict our attention to partitions with $\lambda_{1}=3$. After applying Lemma 13 and Lemma 14, the only partitions remaining are those of the form $\left(2^{f_{2}} 3^{1}\right)$, which are Frobenius, once again, by Lemma 15. Thus, as in the case $d=2$, there is exactly one such Frobenius partition if and only if the weight is odd.

$\mathbf{d}=\mathbf{4}, \mathbf{n} \geqslant \mathbf{1 7}$ : Finally, using the results for $d=1,2$, and 3 , we consider only partitions with $\lambda_{1}=4$. After applying Lemma 13 and Lemma 14, we are left with seven cases to consider.

1. $\left(3^{1} 4^{f_{4}}\right)$ : Partitions of this form are Frobenius by Lemma 15 .

2. $\left(3^{2} 4^{f_{4}}\right)$ : Applying the sequence of moves $\left(F_{v}\right),(P),\left(F_{h}\right),(R),(R),(B),\left(F_{v}\right),(P),\left(F_{h}\right)$ to the corresponding meander results in the meander for a partition of the form $\left(1^{1} f^{f_{4}}\right)$, which is found to be Frobenius in case 5 below.

3. $\left(2^{1} 4^{f_{4}}\right)$ : Applying the sequence of moves $\left(F_{h}\right),\left(F_{v}\right),(P),\left(F_{v}\right),\left(F_{h}\right),(B)$ inductively to the corresponding meander results in the meander for the seaweed algebra of type $\frac{2}{2}$, which has index 1 .

4. $\left(2^{1} 3^{1} 4^{f_{4}}\right)$ : Applying the sequence of moves $\left(F_{v}\right),(P),\left(F_{h}\right),(B),\left(F_{v}\right),(R),(B),\left(F_{h}\right)$ to the corresponding meander results in the meander for a partition of the form $\left(1^{1} 4^{f_{4}}\right)$, which is found to be Frobenius in case 5 below.

5. $\left(1^{1} 4^{f_{4}}\right)$ : Partitions of this form are Frobenius by Lemma 15 .

6. $\left(1^{1} 2^{f_{2}} 4^{f_{4}}\right), f_{2} \geqslant 1$ : Applying the sequence of moves $\left(F_{v}\right),(P),\left(F_{h}\right),(P)$ to the corresponding meander results in a seaweed algebra of type $\frac{2|1| n-8}{2|\cdots| 2|4| \cdots \mid 4}$, which splits into at least two components, $\frac{2}{2}$ and $\frac{1 \mid n-8}{2|\cdots \cdot 2||\cdots| \cdot \mid}$, and is therefore not Frobenius by Theorem 4 . 
7. $\left(1^{2} 4^{f_{4}}\right)$ : Applying the sequence of moves $\left(F_{h}\right),\left(F_{v}\right),(P),(P),\left(F_{v}\right),\left(F_{h}\right),(P),(B),\left(F_{h}\right)$ to the corresponding meander results in the meander for a partition of the form $\left(1^{1} 4^{f_{4}}\right)$, which was found to be Frobenius in case 5 above.

Thus, there are two Frobenius partitions with weight congruent to $1(\bmod 4)$; two with weight congruent to $2(\bmod 4)$; and one with weight congruent to $3(\bmod 4)$.

Remark 17. Similar methods to those used in the proof of Theorem 16 can be used to establish periodic behavior for $d \in\{5,6,7\}$. In the case of $d=5$, the period is of length 4 , while in the cases of $d \in\{6,7\}$, the period jumps to 14 (see Example 18).

Example 18. The sequence of values of $\left|\mathcal{P}_{0}(n, d)\right|$, for $d \in\{5,6,7\}$, along with the value of $n$ at which $\left|\mathcal{P}_{0}(n, d)\right|$ becomes periodic:

$$
\begin{gathered}
d=5, n \geqslant 21: 7,3,5,3 \\
d=6, n \geqslant 37: 14,5,9,3,11,5,11,3,12,5,8,3 \\
d=7, n \geqslant 41: 19,9,18,7,19,9,17,7,20,9,17,7 .
\end{gathered}
$$

Remark 19. At $d=8$ the periodicity stops. This can be seen by considering $\left|\mathcal{P}_{0}(n, 8)\right|$, for $n \equiv 1(\bmod 8)$, where, for $n=8 m+1$, we have that $\operatorname{ind}_{w(\lambda)}\left(1^{1} 4^{2(m-k)} 8^{k}\right)=0$.

\section{Conclusion}

We use the recent index theory of seaweed algebras to generate partition statistics. Starting with a partition $\lambda$, we form a seaweed algebra and define the index of the latter to be the index of the former. Such seaweeds (and attendant index-based statistics) are defined by pairing $\lambda$ with a $\lambda$-based composition. We consider the two extremal cases defined by $\left(\lambda, 1^{w(\lambda)}\right)$ and $(\lambda, w(\lambda))$.

- In studying pairs $\left(\lambda, 1^{w(\lambda)}\right)$ we establish a relation between our new index based statistics and the more classical theory of integer partitions in the form of twocolored partitions.

- As noted in the Introduction, the enumeration of Frobenius seaweeds is of keen topical interest. In studying pairs $(\lambda, w(\lambda))$, we find, remarkably, that the number of such Frobenius pairs is eventually periodic.

This investigation raises more questions and directions for study. We list a few.

1. (Intermediate composition choices) Other "intermediate" $\lambda$-based composition choices might be of interest. For example, $\lambda$ could be paired with its conjugate. It is straightforward to verify that

$$
\left|\left\{\lambda \in \mathcal{P}(n): \operatorname{ind}_{\lambda^{C}}(\lambda)=n-1\right\}\right|
$$

is equal to twice the number of self-conjugate partitions of $n$. 
2. (Congruence relations) Historically, statistics on partitions were motivated by their utility to witness congruence relations $[\mathbf{3}, \mathbf{5}, \mathbf{6}, \mathbf{1 8}]$. Do index-based statistics have similar classical value?

3. (Weighted sums) After the fashion of Euler's Pentagonal Number Theorem [2] and other Legendre type theorems [4], index-based statistics could also be incorporated into weighted sums. Normally in Legendre type theorems, partitions $\lambda$ contribute a term of the form $(-1)^{l(\lambda)} q^{w(\lambda)}$ to the weighted sum, where $l(\lambda)$ is the number of parts of $\lambda$. One could instead insist that each partition contributes a term of the form $(-1)^{\operatorname{ind}_{w(\lambda)}(\lambda)} q^{w(\lambda)}$. For example, by restricting to partitions with only odd parts (denoted $\mathcal{P}\left(n, S_{\text {odd }}\right)$ ), considering the sets

$$
e_{n}=\mid\left\{\lambda \in \mathcal{P}\left(n, S_{\text {odd }}\right): \operatorname{ind}_{w(\lambda)}(\lambda) \text { is even }\right\} \mid
$$

and

$$
o_{n}=\mid\left\{\lambda \in \mathcal{P}\left(n, S_{\text {odd }}\right): \operatorname{ind}_{w(\lambda)}(\lambda) \text { is odd }\right\} \mid,
$$

and insisting that all terms in the weighted sum are non-negative, numerical data suggests the following interesting conjecture.

Conjecture 20.

$$
\sum_{n \geqslant 0}\left|e_{n}-o_{n}\right| q^{n}=\prod_{k \geqslant 1} \frac{1}{1+(-1)^{k} q^{2 k-1}} .
$$

Remark 21. In an attempt to prove Conjecture 20, Seo and Yee [24] established the following

$$
\sum_{n \geqslant 0}(-1)^{\left\lceil\frac{n}{2}\right\rceil}\left(o_{n}-e_{n}\right) q^{n}=\prod_{k \geqslant 1} \frac{1}{1+(-1)^{k} q^{2 k-1}} .
$$

In [7], S. Chern appears to have supplied a proof that $(-1)^{\left\lceil\frac{n}{2}\right\rceil}\left(o_{n}-e_{n}\right)>0$, for $n \geqslant 0$, which completes the proof of Conjecture 20.

\section{References}

[1] G.E. Andrews. "The theory of partitions." No. 2. Cambridge University Press, 1998.

[2] G.E. Andrews. "Euler's pentagonal number theorem." Mathematics Magazine, 56(5): 279-284, 1983.

[3] G.E. Andrews and F.G. Garvan. "Dyson's crank of a partition." Bulletin of the American Mathematical Society, 18(2): 167-171, 1988.

[4] G.E. Andrews and A.J. Yee. "Legendre theorems for subclasses of overpartitions." Journal of Combinatorial Theory, Series A, 144: 16-36, 2016.

[5] A.L. Atkin and P. Swinnerton-Dyer. "Some properties of partitions." Proceedings of the London Mathematical Society, 4: 84-106, 1954. 
[6] F. Breuer, D. Eichhorn, and B. Kronholm. "Polyhedral geometry, supercranks, and combinatorial witnesses of congruences for partitions into three parts." European Journal of Combinatorics, 65: 230-252, 2017.

[7] S. Chern. "Nonmodular infinite products and a Conjecture of Seo and Yee." arXiv:1912.10341, 2019.

[8] W.E. Clark, M. Elhamdadi, X.D. Hou, M. Saito, and T. Yeatman. "Connected quandles associated with pointed abelian groups." Pacific Journal of Mathematics, 264(1): 31-60, 2013.

[9] W.E. Clark and X.D. Hou. "Galkin quandles, pointed abelian groups, and sequence A000712." The Electronic Journal of Combinatorics, 20(1): \#P45, 2013.

[10] V. Coll, A. Dougherty, M. Hyatt, and N. Mayers. "Meander graphs and Frobenius seaweed algebras III." Journal of Generalized Lie Theory and Applications, 11(2), 2017.

[11] V. Coll, A. Giaquinto, and C. Magnant. "Meanders and Frobenius seaweed Lie algebras." Journal of Generalized Lie Theory and Applications, 5, 2011.

[12] V. Coll, M. Hyatt, C. Magnant, and H. Wang. "Meander graphs and Frobenius seaweed Lie algebras II." Journal of Generalized Lie Theory and Applications, 9(1), 2015.

[13] V. Coll, C. Magnant, and H. Wang. "The signature of a meander." arXiv:1206.2705, 2012.

[14] V. Coll, A. Mayers, and N. Mayers. "Statistics on partitions arising from seaweed algebras." arXiv:1809.09271v2, 2018.

[15] V. Dergachev and A. Kirillov. "Index of Lie algebras of seaweed type." Journal of Lie Theory, 10(2): 331-343, 2000.

[16] J. Dixmier. "Enveloping Algebras." Vol. 14. Newnes, 1977.

[17] M. Duflo and R. Yu. "On compositions associated to Frobenius parabolic and seaweed subalgebras of $\mathfrak{s l}_{n}(k)$." Journal of Lie Theory, 25: 1191-1213, 2015.

[18] F.J. Dyson. "Some guesses in the theory of partitions." Eureka, 8(10): 10-15, 1944.

[19] M. Gerstenhaber and A. Giaquinto. "Boundary solutions of the classical Yang-Baxter equation." Letters in Mathematical Physics, 40(4): 337-353, 1997.

[20] M. Gerstenhaber and A. Giaquinto. "Graphs, Frobenius functionals, and the classical Yang-Baxter equation." arXiv:0808.2423v1, August 18, 2008.

[21] H. Gupta. "Tables of partitions." Vol. 4. Published for the Royal Society at the University Press, 1958.

[22] A. Ooms. "On Lie algebras having a primitive universal enveloping algebra." Journal of Algebra, 32(3): 488-500, 1974.

[23] S. Ramanujan. "Some properties of $p(n)$, the number of partitions of $n$." Proc. Cambridge Phil. Soc. Vol. 19. 1919.

[24] S. Seo and A.J. Yee. "Index of seaweed algebras and integer partitions." The Electronic Journal of Combinatorics, 27(1): \#P1.47, 2020. 